Publ. RIMS, Kyoto Univ.

38 (2002), 725-733

\title{
Quasianalyticity of Positive Definite Continuous Functions
}

\author{
By \\ Soon-Yeong CHUNG*
}

\begin{abstract}
It is shown that for a positive definite continuous function $f(x)$ on $\mathbb{R}^{n}$ the followings are equivalent:

(i) $f(x)$ is quasaianalytic in some neighborhood of the origin.

(ii) $f(x)$ can be expressed as an integral $f(x)=\int_{\mathbb{R}_{n}} e^{i x \xi} d \mu(\xi)$ for some positive Radon measure $\mu$ on $\mathbb{R}^{n}$ such that $\int \exp M(L|\xi|) d \mu(\xi)$ is finite for some $L>0$ where the function $M(t)$ is a weight function corresponding to the quasaianalyticity.
\end{abstract}

(iii) $f(x)$ is quasaianalytic everywhere in $\mathbb{R}^{n}$.

Moreover, an analogue for the analyticity is also given as a corollary.

\section{$\S 1 . \quad$ Introduction}

A continuous function $f(x)$ on $\mathbb{R}^{n}$ is said to be positive definite if

$$
\sum_{j, k=1}^{m} f\left(x_{j}-x_{k}\right) \zeta_{j} \overline{\zeta_{k}} \geq 0
$$

for any $x_{1}, x_{2}, \ldots, x_{m} \in \mathbb{R}^{n}$ and $\zeta_{1}, \zeta_{2}, \ldots, \zeta_{m} \in \mathbb{C}$.

It is well known that if a continuous function $f(x)$ on $\mathbb{R}^{n}$ which is positive definite belongs to $C^{2 k}$ in a neighborhood of the origin then $f(x)$ belongs to

Communicated by T. Kawai. Received March 30, 2001. Revised December 18, 2001.

2000 Mathematics Subject Classification(s): Primary 42A82; Secondary 46F05.

Key words and phrases: positive definite, quasianalyticity.

This paper was supported by grant No.1999-2-101-001-5 from KOSEF.

*Department of Mathematics, Sogang University, Seoul 121-742, Korea.

e-mail: sychung@ccs.sogang.ac.kr 
$C^{2 k}$ everywhere in $\mathbb{R}^{n}$ (see [D, p. 186]). This can be proved by the advantage of Bochner's theorem: every positive definite continuous function is the Fourier (inverse) transform of a positive Radon measure of finite total mass. In fact, a similar argument for the analyticity is easily derived from the well-known theorems for general positive semidefinite analytic kernels (see $[B]$ and $[F]$ ). Besides, the non-quasianalytic ultradifferentiability was also considered in the paper [CI].

The quasaianalyticity is an intermediate regularity of functions between differentiability and analyticity which is closer to analyticity than the other (see $[\mathrm{K}])$.

Thus, it will be quite interesting to investigate the quasaianalyticity for positive definite continuous functions.

In this paper we show that the following three conditions are equivalent for a positive definite continuous function $f(x)$ on $\mathbb{R}^{n}$ :

(i) $f(x)$ is quasaianalytic in some neighborhood of the origin.

(ii) $f(x)$ can be expressed as an integral $f(x)=\int_{\mathbb{R}^{n}} e^{i x \xi} d \mu(\xi)$ for some positive Radon measure $\mu$ on $\mathbb{R}^{n}$ such that $\int \exp M(L|\xi|) d \mu(\xi)$ is finite for some $L>0$ where the function $M(t)$ is a weight function corresponding to the quasaianalyticity.

(iii) $f(x)$ is quasaianalytic everywhere in $\mathbb{R}^{n}$.

A parallel result for the analyticity is also given as a corollary. We shall prove these results in an unified way, which includes the analyticity, quasianalyticity, non-quasianalytic ultradifferentiability and so on, simultaneously.

\section{$\S 2 . \quad$ Notations and Preliminaries}

Throughout this paper we use a conventional multi-index notation such as $|\alpha|=\alpha_{1}+\alpha_{2}+\cdots+\alpha_{n}, \partial^{\alpha}=\partial_{1}^{\alpha_{1}} \partial_{2}^{\alpha_{2}} \cdots \partial_{n}^{\alpha_{n}}, \partial_{j}=\partial / \partial x_{j}$ for $\alpha \in \mathbb{N}_{0}^{n}$ where $\mathbb{N}_{0}^{n}$ is the set of all nonnegative integers.

First, we introduce the ultradifferentiable class including the analytic class, quasianalytic class, and non-quasianalytic class.

Let $\left(M_{p}\right)_{p=0}^{\infty}$ be a sequence of positive numbers satisfying the following conditions:

(M.0) There exist constants $C>0$ and $H>0$ such that

$$
p ! \leq C H^{p} M_{p}, \quad p=0,1,2, \ldots
$$


(M.1) $M_{p}^{2} \leq M_{p-1} M_{p+1}, p=1,2, \ldots$.

(M.2) $)^{\prime}$ There exist $A>0$ and $B>0$ such that

$$
M_{p+1} \leq A B^{p} M_{p}, \quad p=0,1,2, \ldots .
$$

For an open subset $\Omega$ of $\mathbb{R}^{n}$ and a sequence $M_{p}$ as above we denote by $\mathcal{E}^{\left\{M_{p}\right\}}(\Omega)\left(\mathcal{E}^{\left(M_{p}\right)}(\Omega)\right.$, respectively) the set of all $\phi \in C^{\infty}(\Omega)$ such that for any compact subset $K$ of $\Omega$ there exist constants $h>0$ and $C>0$ (for any $h>0$ there exists a constant $C>0$ ) such that

$$
\sup _{x \in K}\left|\partial^{\alpha} \phi(x)\right| \leq C h^{|\alpha|} M_{|\alpha|}, \quad \alpha \in \mathbb{N}_{0}^{n} .
$$

An element of $\mathcal{E}^{\left\{M_{p}\right\}}(\Omega)\left(\mathcal{E}^{\left(M_{p}\right)}(\Omega), \quad\right.$ respectively) is called $\left\{M_{p}\right\}$ ultradifferentiable $\left(\left(M_{p}\right)\right.$-ultradifferentiable, respectively) function in $\Omega$.

For an instance, $\mathcal{E}^{\left\{p !^{s}\right\}}(\Omega)$ is known as the set of Gevrey functions of index $s$. In particular, if $s=1$ then $\mathcal{E}^{\{p !\}}(\Omega)$ is the set of all (real) analytic functions in $\Omega$ and $\mathcal{E}^{(p !)}(\Omega)$ is set of all analytic functions which extend to $\mathbb{C}^{n}$ as entire functions.

Remark. (i) The conditions (M.0), (M.1), and (M.2)' are the most fundamental and essential in a sense that the sequence $M_{p}$ can be rearranged without any change of $\mathcal{E}^{*}(\Omega)$ so that (M.1) should be satisfied and (M.2) makes $\mathcal{E}^{*}(\Omega)$ stable under the differentiation (see $\left.[\mathrm{K}]\right)$. Here ${ }^{*}$ denotes $\left\{M_{p}\right\}$ or $\left(M_{p}\right)$. Moreover, (M.0) means that the analytic class is the smallest class to be considered here.

(ii) If the sequence $M_{p}$ satisfies $\sum_{p=1}^{\infty}\left(M_{p-1} / M_{p}\right)=\infty$ then an element $\phi$ in $\mathcal{E}^{*}(\Omega)$ with $\partial^{\alpha} \phi\left(x_{0}\right)=0$ for all $\alpha \in \mathbb{N}_{0}^{n}$ is identically zero in a connected open set containing $x_{0}$. In this case every $\phi$ in $\mathcal{E}^{*}(\Omega)$ is said to be quasianalytic in $\Omega$. But if $\sum_{p=1}^{\infty}\left(M_{p-1} / M_{p}\right)<\infty$ then $\mathcal{E}^{*}(\Omega)$ contains the cut-off functions and the partitions of unity.

For a sequence $M_{p}$ as above we define its associated function $M(t)$ on $(0, \infty)$ by

$$
M(t)=\sup _{p} \log \frac{t^{p} M_{0}}{M_{p}} .
$$

Then we note that $M(t)$ is increasing and

$$
M_{p}=M_{0} \sup _{t>0} \frac{t^{p}}{\exp M(t)}
$$

by the virtue of (M.1) (see [K, p. 49]). 


\section{§3. Main Results}

A continuous function $f(x)$ on $\mathbb{R}^{n}$ is said to be positive definite if for any $m \in \mathbb{N}_{0}$

$$
\sum_{j, k} f\left(x_{j}-x_{k}\right) \zeta_{j} \overline{\zeta_{k}} \geq 0
$$

for any $x_{1}, x_{2}, \ldots, x_{m} \in \mathbb{R}^{n}$ and complex numbers $\zeta_{1}, \zeta_{2}, \ldots, \zeta_{m}$.

It is well known as so called Bochner theorem (see [GV]) that every continuous function $f(x)$ on $\mathbb{R}^{n}$ which is positive definite can be expressed as

$$
f(x)=\int e^{i x \xi} d \mu(\xi)
$$

for some positive Radon measure $\mu$ of finite total mass.

As for the smoothness of positive definite continuous function $f(x)$ it is well known that if $f(x)$ is $C^{\infty}$ near the origin then it is $C^{\infty}$ everywhere in $\mathbb{R}^{n}$ (see $[\mathrm{D}]$ ). This makes it possible to expect that the local regularity implies the global regularity. Thus, it will be interesting to consider a regularity of analyticity, quasianalyticity, and so on, from the same point of view. But little has been known so far about such regularity for positive definite continuous functions. The following answers these problems affirmatively:

Theorem 3.1. Let $f(x)$ be a continuous function on $\mathbb{R}^{n}$ which is positive definite. Then the followings are equivalent:

(i) $f(x)$ is $\left\{M_{p}\right\}$-ultradifferentiable in a neighborhood of the origin.

(ii) $f(x)$ has an integral representation

$$
f(x)=\int_{\mathbb{R}^{n}} e^{i x \xi} d \mu(\xi)
$$

with a positive Radon measure $\mu$ satisfying that there exist constants $H>$ 0 and $C>0$ such that

$$
\int\left(1+|\xi|^{2}\right)^{k} d \mu(\xi) \leq C H^{2 k} M_{2 k}, \quad k \in \mathbb{N}_{0}^{n} .
$$

(iii) $f(x)$ has an integral representation as (3.2) with a positive Radon measure $\mu$ satisfying that

$$
\int_{\mathbb{R}^{n}} \exp M(L|\xi|) d \mu<\infty
$$


for some constant $L>0$, where $M(t)$ is the associated function of the sequence $M_{p}$.

(iv) $f(x)$ is $\left\{M_{p}\right\}$-ultradifferentiable everywhere in $\mathbb{R}^{n}$.

Proof. (i) $\Longrightarrow($ ii): In view of the Bochner theorem $f(x)$ can be expressed as

$$
f(x)=\int e^{i x \xi} d \mu(\xi), \quad x \in \mathbb{R}^{n}
$$

with a positive Radon measure $\mu$ of finite total mass. Since $f(x)$ is $C^{\infty}$ near the origin it is already $C^{\infty}$ in $\mathbb{R}^{n}$.

Let $k \in \mathbb{N}_{0}$ and consider a $C^{\infty}$ function $g_{k}(x)$ defined by

$$
g_{k}(x)=(1-\triangle)^{k} f(x), \quad x \in \mathbb{R}^{n}
$$

where $\triangle$ is the Laplace operator. Then by the assumption (i) there exists an open ball $B(0 ; R)$ with its center at 0 and of radius $R$ such that

$$
\left|g_{k}(x)\right| \leq C H^{2 k} M_{2 k}, \quad x \in B(0 ; R), \quad k \in \mathbb{N}_{0}^{n}
$$

for some $C>0$ and $H>0$.

Now we regard $g_{k}(x)$ as a tempered distribution and regularize with a positive regularizing function in $C^{\infty}$ functions with compact support which is also positive definite. This can be done by taking the usual regularizing function $\phi(x)$ which is $C^{\infty}$ and passing to $\psi(x)=\phi * \overline{\phi(-x)}$, which is positive and positive definite simultaneously. Then $\psi_{j}(x)=j^{n} \psi(j x)$ regularizes $g_{k}$ with a sequence $g_{k} * \psi_{j}(x)$.

Then for $x \in B(0 ; R / 2)$ and $j>2 / R$ it follows from (3.5) that

$$
\begin{aligned}
\left|g_{k} * \psi_{j}(x)-g_{k}(x)\right| & =\int\left|g_{k}(x-y)-g_{k}(x)\right| \psi_{j}(y) d y \\
& \leq \int_{|y| \leq R / 2}\left|g_{k}(x-y)-g_{k}(x)\right| \psi_{j}(y) d y \\
& \leq 2 C H^{2 k} M_{2 k}, \quad k \in \mathbb{N}_{0}^{n} .
\end{aligned}
$$

Thus we have

$$
\left|g_{k} * \psi_{j}(x)\right| \leq 3 C H^{2 k} M_{2 k}, \quad k \in \mathbb{N}_{0}^{n}
$$

for any $x \in B(0 ; R / 2)$ and $j>2 / R, k \in \mathbb{N}_{0}^{n}$.

Taking the Fourier transform to $g_{k}$ as a tempered distribution we have

$$
\begin{aligned}
\hat{g}_{k}(\xi) & =\left(1+|\xi|^{2}\right)^{k} \hat{f}(\xi) \\
& =\left(1+|\xi|^{2}\right)^{k} \mu, \quad \xi \in \mathbb{R}^{n} .
\end{aligned}
$$


Then combining the above we have

$$
\begin{aligned}
3 C H^{2 k} M_{2 k} & \geq\left|g_{k} * \psi_{j}(0)\right| \\
& =\left|\int g_{k}(x) \psi_{j}(-x) d x\right| \\
& =\left|\int \hat{g}_{k}(\xi) \hat{\psi}(\xi / j) d \xi\right| \\
& =\int\left(1+|\xi|^{2}\right)^{k} \hat{\psi}(\xi / j) d \mu(\xi) .
\end{aligned}
$$

Thus, applying Fatou's lemma we obtain

$$
\int\left(1+|\xi|^{2}\right)^{k} d \mu(\xi) \leq C H^{2 k} M_{2 k}, \quad k=0,1,2, \ldots
$$

with a new constant $C>0$, which gives (ii).

(ii) $\Longrightarrow($ iii): First, it is easy to see that

$$
\int|\xi|^{2 k} d \mu(\xi) \leq C H^{2 k} M_{2 k}, \quad k=0,1,2, \ldots
$$

Then it follows from (M.1) and (M.2)' that

$$
\begin{aligned}
\int|\xi|^{2 k+1} d \mu(\xi) & \leq \int\left(|\xi|^{2 k}+|\xi|^{2(k+1)}\right) d \mu(\xi) \\
& \leq C H^{2 k} M_{2 k}+C H^{2(k+1)} M_{2 k+2} \\
& \leq \frac{C M_{0}}{H M_{1}} H^{2 k+1} M_{2 k+1}+C A H(B H)^{2 k+1} M_{2 k+1} \\
& \leq C_{1} H_{1}^{2 k+1} M_{2 k+1}, \quad k=0,1,2, \ldots
\end{aligned}
$$

with new constants $C_{1}>0$ and $H_{1}>0$. Thus we have

$$
\int|\xi|^{k} d \mu(\xi) \leq C H^{k} M_{k}, \quad k=0,1,2, \ldots
$$

for some constants $C>0$ and $H>0$.

Now consider a sequence $m_{p}$ defined by

$$
m_{p}=\frac{M_{p}}{M_{p-1}}, \quad p=1,2,3, \ldots .
$$

Then (M.0) and (M.1) imply that $m_{p}$ increases to $\infty$ as $p \longrightarrow \infty$.

Now let $L=1 /(2 H)$ where $H$ is the constant in (3.8) and for each $\xi \in \mathbb{R}^{n}$ choose an integer $q(\xi)>0$ such that

$$
m_{q(\xi)} \leq L|\xi|<m_{q(\xi)+1} .
$$


Then it follows that for any $\xi \in \mathbb{R}^{n}$

$$
\begin{aligned}
M(L|\xi|) & =\sup _{p} \log \frac{M_{0}(L|\xi|)^{p}}{M_{p}} \\
& =\sup _{p} \log \left(\prod_{k=1}^{p} \frac{L|\xi|}{m_{k}}\right) \\
& =\log \left(\prod_{k=1}^{q(\xi)} \frac{L|\xi|}{m_{k}}\right) \\
& =\log \frac{M_{0}(L|\xi|)^{q(\xi)}}{M_{q(\xi)}} .
\end{aligned}
$$

Consequently, from (3.8) we have

$$
\begin{aligned}
\int \exp M(L|\xi|) d \mu(\xi) & =\int \frac{M_{0}(L|\xi|)^{q(\xi)}}{M_{q(\xi)}} d \mu(\xi) \\
& \leq \int \sum_{p=0}^{\infty} \frac{M_{0}(L|\xi|)^{p}}{M_{p}} d \mu(\xi) \\
& =\sum_{p=0}^{\infty} \frac{M_{0}}{2^{p}} \int \frac{|\xi|^{p}}{H^{p} M_{p}} d \mu(\xi) \\
& \leq C M_{0} \sum_{p=0}^{\infty} \frac{1}{2^{p}}
\end{aligned}
$$

which is finite. This proves (iii).

(iii) $\Longrightarrow\left(\right.$ iv): For any $x \in \mathbb{R}^{n}$ and $\alpha \in \mathbb{N}_{0}^{n}$ we have

$$
\begin{aligned}
\left|\partial^{\alpha} f(x)\right| & =\left|\int e^{i x \xi}(i \xi)^{\alpha} d \mu(\xi)\right| \\
& \leq H^{|\alpha|} M_{|\alpha|} \int \frac{|\xi|^{|\alpha|}}{H^{|\alpha|} M_{|\alpha|}} d \mu(\xi) \\
& \leq \frac{H^{|\alpha|} M_{|\alpha|}}{M_{0}} \int \sup _{\alpha \in \mathbb{N}_{0}^{n}} \frac{M_{0}|\xi|^{|\alpha|}}{H^{|\alpha|} M_{|\alpha|}} d \mu(\xi) \\
& \leq \frac{H^{|\alpha|} M_{|\alpha|}}{M_{0}} \int \exp M(|\xi| / H) d \mu(\xi) \\
& \leq C_{0} H^{|\alpha|} M_{|\alpha|}
\end{aligned}
$$

by taking $H=1 / L$ in (3.4) and for some $C_{0}>0$. This implies that $f(x)$ is $\left\{M_{p}\right\}$-ultradifferentiable everywhere in $\mathbb{R}^{n}$.

(iv) $\Longrightarrow(\mathrm{i})$ : it is trivial. 
Remark. (i) In the proof of (iii) $\Longrightarrow$ (iv) we can see that each derivative of $f(x)$ is bounded on $\mathbb{R}^{n}$. In fact, we proved much better statement than the fact that $f(x)$ is only $\left\{M_{p}\right\}$-ultradifferentiable in $\mathbb{R}^{n}$, by the inequality

$$
\sup _{x \in \mathbb{R}^{n}}\left|\partial^{\alpha} f(x)\right| \leq C H^{|\alpha|} M_{|\alpha|} .
$$

In particular, if $M_{p}=p$ ! then this inequality implies that $f(x)$ can be extended to a tubular neighborhood of $\mathbb{R}^{n}$ in $\mathbb{C}^{n}$ as a holomorphic function there.

(ii) The above theorem excludes neither the quasianalytic class nor the non-quasaianalytic class.

(iii) In the proof above we actually proved that (3.8) is also equivalent to (3.3) and (3.4) respectively.

Concerning the analyticity it is easy to derive a similar argument for the positive definite continuous functions by taking advantage of the well-known theorems for general positive semidefinite analytic kernels (see $[\mathrm{B}]$ and $[\mathrm{F}]$ ). Nevertheless, the above theorem does not exclude the analytic case. Hence we restate the analytic version as a corollary as follows:

Corollary 3.2. Let $f(x)$ be a continuous function on $\mathbb{R}^{n}$ which is positive definite. Then the followings are equivalent:

(i) $f(x)$ is analytic in a neighborhood of the origin.

(ii) $f(x)$ has an integral representation as (3.2) with a positive Radon measure $\mu$ satisfying that there exist constants $H>0$ and $C>0$ such that

$$
\int\left(1+|\xi|^{2}\right)^{k} d \mu(\xi) \leq C H^{2 k}(2 k) !
$$

for all $k=0,1,2, \ldots$.

(iii) $f(x)$ has an integral representation as (3.2) for a positive Radon measure $\mu$ satisfying that

$$
\int \exp L|\xi| d \mu(\xi)<\infty
$$

for some constant $L>0$.

(iv) $f(x)$ is analytic everywhere in $\mathbb{R}^{n}$.

As for the $\left(M_{p}\right)$-ultradifferentiable class a parallel result can be proved in a similar way as in Theorem 3.1, with only a slight modification of the quantifiers. Thus we state it without proof. 
Theorem 3.3. Let $f(x)$ be a positive definite continuous function on $\mathbb{R}^{n}$. Then the followings are equivalent:

(i) $f(x)$ is $\left(M_{p}\right)$-ultradifferentiable in a neighborhood of the origin.

(ii) $f(x)$ has an integral representation as (3.2) with a positive Radon measure $\mu$ satisfying that for any $h>0$ there exists a constant $C>0$ such that

$$
\int\left(1+|\xi|^{2}\right)^{k} d \mu(\xi) \leq C h^{2 k} M_{2 k}
$$

for $k=0,1,2, \ldots$.

(iii) $f(x)$ has an integral representation as (3.2) with a positive Radon measure $\mu$ satisfying that for any $L>0$

$$
\int \exp M(L|\xi|) d \mu(\xi)<\infty
$$

(iv) $f(x)$ is $\left(M_{p}\right)$-ultradifferentiable everywhere in $\mathbb{R}^{n}$.

\section{Acknowledgement}

Author would like to express his deep gratitude to anonymous referee for reminding him of the references $[\mathrm{B}]$ and $[\mathrm{F}]$ from which he could see that the analyticity version can be easily derived.

\section{References}

[B] Bremermann, H., Holomorphic continuation of kernel function and the Bergman metric in several complex variables, Univ. Michigan Press, Ann Arbor, 1955.

[C] Chung, S.-Y., Positive definite temperature functions and a correspondence to positive temperature functions, Proc. Royal Soc. Edinburgh, 127A (1997).

[CCK] Chung, J., Chung, S.-Y. and Kim, D., Positive definite hyperfunctions, Nagoya Math. J., 140 (1995), 139-149.

[CI] Chung, S.-Y. and Yim, S.-Y., Global regularity of positive definite continuous functions Integral Transform. Spec. Funct., 11 (2000), 345-352.

[CK] Chung, S.-Y. and Kim, D., Distributions with exponential growth and BochnerSchwartz theorem for Fourier hyperfunctions, Publ. RIMS, Kyoto Univ., 31 (1995), 829-845.

[D] Donoghue, W. F., Distributions and Fourier Transforms, Academic Press, New York, 1969.

[F] Fitzgerald, C. H., On analytic continuation to Schlicht function, Proc. Amer. Math. Soc., 18 (1967), 788-792.

[GHS] Guo, K., Hu, S. and Sun, X., Conditionally positive definite functions and LaplaceStieltjes integrals, J. Approx. Theory, 74 (1993), 249-265.

[GV] Gelfand, I. M. and Vilenkin, N. Y., Generalized Functions, Academic Press, New York, 1964.

[K] Komatsu, H., Ultradistributions I; Structure theorem and a characterization, J. Fac. Sci. Univ. Tokyo. Sect. IA, 20 (1973), 25-105.

[MN] Madych, W. R. and Nelson, S. A., Multivariate interpolation and conditionally positive definite functions II, Math. Comp., 54 (1990), 211-230. 